\title{
O impacto da pandemia de Covid-19 na saúde mental e física de crianças e adolescentes: uma revisão narrativa
}

\author{
The impact of the Covid-19 pandemic on the mental and physical health of children and \\ adolescents: a narrative review \\ El impacto de la pandemia de Covid-19 en la salud física y mental de niños y \\ adolescentes: una revisión narrativa
}

Danielle Braz Amarílio da Cunha ${ }^{1 *}$, Anna Beatriz Sanguinetti Regadas de Barros ${ }^{1}$, Juliana Barrozo Fernandes Borges ${ }^{1}$, Larissa Müller Marques ${ }^{1}$, Marília Magalhães Wanderlei ${ }^{1}$, Vítor Henrique Soares Campelo ${ }^{1}$, Danielle Sampaio Lima da Cruz ${ }^{2}$.

\section{RESUMO}

Objetivo: Revisar e descrever os impactos da pandemia da doença por coronavírus 2019 (COVID-19) nas crianças e adolescentes em seu cotidiano, saúde mental e física, abordando especialmente a perspectiva do isolamento social. Revisão bibliográfica: A pandemia da COVID-19 provocou diversas consequências sociais, econômicas, psicológicas e físicas, especialmente em populações mais vulneráveis como as crianças e os adolescentes. Observou-se mudanças desde os hábitos alimentares, até os padrões de saúde mental. Desse modo, o estudo optou por selecionar cinco principais impactos mencionados na literatura, como o uso de telas, o fechamento das escolas, a saúde mental, a alimentação e a violência doméstica. Considerações finais: O cotidiano das crianças e adolescentes foi significativamente afetado com a pandemia da COVID-19, devido a problemas financeiros e de saúde enfrentados por familiares. Nesse sentido, é de suma importância atentar-se à tal população nesse momento tão delicado, a fim de amenizar os impactos da pandemia em suas vidas.

Palavras-chave: Isolamento social, Pediatria, Atividades cotidianas.

\section{ABSTRACT}

Objective: Review and describe the impacts of the coronavirus disease 2019 (COVID-19) pandemic on children and adolescents in their daily lives, mental and physical health, approaching, especially, from the perspective of social isolation. Literature review: The COVID-19 pandemic has had several social, economic, psychological and physical consequences, especially in the most vulnerable populations such as children and adolescents. Changes were observed from eating habits to mental health patterns. Along these lines, the study chose to select five main impacts mentioned in the literature, such as the use of screens, school closings, mental health, food and domestic violence. Final considerations: The daily lives of children and adolescents were significantly affected with COVID-19 pandemic, because of financial and health problems faced by family members. In this way, it is very necessary to pay attention to this population at this delicate moment, aiming to alleviate the impacts of the pandemic on their lives.

Key words: Social isolation, Pediatrics, Activities of daily living.

\section{RESUMEN}

Objetivo: Revisar y describir los impactos de la pandemia enfermedad por coronavirus 2019 (COVID-19) en los niños y adolescentes en su vida diaria, salud mental y física, abordando especialmente la perspectiva del aislamiento social. Revisión bibliográfica: La pandemia de COVID-19 ha tenido varias consecuencias sociales, económicas, psicológicas y físicas, especialmente en las poblaciones más vulnerables como los niños y adolescentes. Se observaron cambios desde los hábitos alimenticios, hasta los patrones de salud mental. Así, el estudio optó por seleccionar cinco impactos principales mencionados en la literatura: (1) uso

${ }^{1}$ Centro Universitário de Brasília (UniCEUB), Brasília - DF. *E-mail: danielle.brazac@gmail.com

2 Universidade Católica de Brasília (UCB), Brasília - DF. 
de pantallas, (2) cierre de escuelas, (3) salud mental, (4) alimentación y (5) violencia doméstica. Consideraciones finales: La vida cotidiana de los niños y adolescentes se vio significativamente afectada por la pandemia COVID-19, debido a problemas económicos y de salud que enfrentan los miembros de la familia. Por ello, es necesario prestar atención a esta población en este delicado momento, con el objetivo de paliar los impactos de la pandemia en sus vidas.

Palabras clave: Aislamiento social, Pediatría, Actividades cotidianas.

\section{INTRODUÇÃO}

Em dezembro de 2019, surgia em Hubei, uma província Chinesa, casos de pneumonia cuja etiologia era desconhecida. Posteriormente, descobriu-se que o causador da doença era um vírus da família Coronaviridae, denominado Coronavírus 2 da síndrome respiratória aguda grave (SARS-CoV-2), causador da doença por coronavírus 2019 (COVID-19). A doença alcançou dimensões tão significativas que em março de 2020, a Organização Mundial da Saúde (OMS) a declarou como uma pandemia (PEDROSA AL, et al., 2020; WORLD HEALTH ORGANIZATION (WHO), 2020).

No Brasil, o primeiro registro da doença se deu em fevereiro de 2020. A partir de então, a sociedade ficou em alerta e teve que mudar drasticamente seus hábitos. Não apenas em território brasileiro, mas quase todos os países do globo iniciaram medidas para retardar a transmissão do novo vírus, como recomendações de ficar em casa, lockdown (confinamento) e toque de recolher (PEDROSA AL, et al., 2020). Apenas atividades consideradas essenciais, como mercados e farmácias, continuaram funcionando e os sentimentos de medo e insegurança se instalaram em todos (FOLINO CH, et al., 2021).

Tais medidas provocaram impactos em toda sociedade, tanto em sua vida cotidiana, como, de um ponto de vista mais abrangente, em sua saúde física e mental. Nesse sentido, acredita-se que o confinamento possa provocar sintomatologias depressivas, insônia, irritabilidade, exaustão emocional e outros problemas de saúde mental (VALERO ALZAGA E, et al., 2020). Ademais, tem-se a preocupação com a possibilidade de ser infectado ou de familiares se infectarem, preocupações financeiras, bem como mudanças no estilo de vida, no padrão alimentar e de peso (FERNÁNDEZ-ARANDA F, et al., 2020).

Esses impactos não pouparam a população pediátrica, que também se encontra entre os afetados com as restrições da pandemia de COVID-19, especialmente por serem mais vulneráveis. Embora as crianças sejam apontadas como o grupo menos afetado pelos sintomas da infecção, elas não estão ilesas à doença e suas formas graves e, além disso, elas sofrem com as consequências das medidas de contenção da pandemia. Com o isolamento social, por exemplo, seu cotidiano foi afetado de várias formas, incluindo a interrupção da vida escolar presencial, problemas financeiros e de saúde nas famílias (LEVANDOWSKI ML, et al., 2021).

Nesse sentido, não obstante o distanciamento social seja fundamental para diminuir a propagação exponencial do vírus, muitas crianças e adolescentes podem estar enfrentando uma situação de risco aumentado: os casos de violência doméstica contra crianças e adolescentes têm aumentado significativamente, o uso da internet tem se tornado excessivo, o número de crianças e adolescentes que desenvolveram obesidade recrudesceu consideravelmente, além dos inúmeros distúrbios psicológicos, como depressão e ansiedade, gerados por esse isolamento social (NOGUEIRA-DE-ALMEIDA CA, et al., 2020).

Desse modo, é importante se atentar às crianças nesse momento tão delicado para tentar amenizar os impactos de toda essa pandemia em sua saúde tanto física quanto mental (FIGUEIREDO CS, et al., 2021). Portanto, o presente estudo objetivou analisar os impactos do isolamento social nas diferentes vertentes da vida de crianças e adolescentes e a repercussão de tais consequências em seu cotidiano.

\section{REVISÃO BIBLIOGRÁFICA}

A pandemia da COVID-19 provocou diversas consequências, sendo visível o impacto dessa situação, principalmente, em populações mais vulneráveis, como as crianças, as quais foram afetadas em níveis biológicos, socioeconômicos e psicológicos. Essas populações passaram a ser expostas a obstáculos, como: 
acesso reduzido a alimentos, a serviços sociais e de saúde, fechamento prolongado de escolas, entre outros (GRABER KM, et al., 2021). Além disso, outras atividades sofreram mudanças com a pandemia, como a diminuição e interrupção de relações sociais e atividades físicas, resultando em alterações de hábitos alimentares e do ritmo circadiano. Dessa forma, é possível observar como o isolamento e as restrições se tornaram um grande desafio para essa população (MELEGARI MG, et al., 2021).

Portanto, tendo em vista todos os cuidados instituídos, é perceptível uma mudança nos hábitos de vida dessa população, como redução de atividades ao ar livre, e, consequentemente, maior tempo de exposição às telas, associado a outros fatores, como a alimentação inadequada e sedentarismo. (FIGUEIREDO CS, et al., 2021)

Na esfera da saúde mental, estudos indicam que, durante e depois do isolamento, crianças e adolescentes tornaram-se mais propensos a desenvolver quadros depressivos e ansiosos, decorrentes da solidão que experimentaram durante a quarentena (SEGRE G, et al., 2021). Ademais, a mudança do ensino tradicional presencial para o ensino a distância, por ser um evento inesperado, também provocou impactos em diferentes aspectos das vidas das crianças (MISIRLI O e ERGULEC F, 2021).

Outrossim, o contexto induzido pela pandemia da COVID-19 modificou os hábitos alimentares e o estilo de vida das famílias. Observou-se desde a falta de alimentos, principalmente nas famílias de baixa renda, até impactos associados ao fechamento de escolas, as quais possuíam programas que disponibilizavam merenda escolar gratuita, de forma que isso fosse interrompido pelo isolamento. Dessarte, sabe-se que houve um comprometimento da quantidade, qualidade e diversidade da dieta dessas crianças, podendo contribuir tanto para desnutrição, quanto para obesidade (ZEMRANI B, et al., 2021). Além disso, estudos apontam que o isolamento social predispõe a uma maior motivação para consumir alimentos gordurosos e saborosos, com a finalidade de aliviar efeitos negativos que podem ocorrer dentro desse contexto, elevando as chances de do desenvolvimento da obesidade (FIGUEIREDO CS, et al., 2021).

Por fim, outro impacto da pandemia no cotidiano de crianças e adolescentes fora o aumento das taxas de violência doméstica. Isso porque tanto as crianças, quanto os adolescentes passaram a ficar mais tempo confinados com seus agressores, refletindo no aumento nos números de agressões físicas e psicológicas. Ademais, o fechamento das escolas impossibilita que os professores e profissionais da rede de ensino reconheçam sinais de violência praticados contra os jovens fora do ambiente escolar (LEVANDOWSKI ML, et al., 2021).

Nesse sentido, para melhor compreender as principais áreas da vida de crianças e adolescentes afetadas pela pandemia da COVID-19, este estudo listou cinco principais fatores que estariam envolvidos, buscando compreendê-los em sua forma integral, sendo eles: uso de telas durante a quarentena, saúde mental, impactos do fechamento das escolas, alimentação e violência doméstica.

\section{Uso de telas durante a quarentena}

Devido a pandemia, as crianças foram proibidas de brincar fora de casa, seja na rua, nos parques ou qualquer outra atividade ao ar livre. Isso levou a um aumento da exposição dos jovens à tela de computadores e celulares. Com isso, alguns fatores negativos foram constatados, como aumento do consumo de comidas não saudáveis, diminuição dos exercícios físicos, aumento de problemas físicos e prejuízo no aprendizado (FIGUEIREDO CS, et al., 2020).

O uso maciço dos computadores e celulares levou também a uma dependência da internet por parte das crianças. O estudo fica prejudicado pela fácil forma de se buscar informação, além também de gerar uma provável dificuldade de reabilitação social quando a pandemia acabar (DESLANDES SF e COUTINHO T, 2020). Observou-se também, maior desregulação do sono das crianças, as quais passaram a ter menor quantidade de sono de boa qualidade durante os dias, levando a um cansaço e uma indisposição elevados (IMRAN N, et al., 2020).

Foi relatado também um aumento da ansiedade nas crianças, gerando assim pensamentos mais negativos e conflituosos que podem levar a uma repercussão ainda mais grave como depressão ou até mesmo em pensamentos suicidas (LARSEN L, et al., 2021). 
Ademais, a maior exposição ao uso de telas levou, consequentemente, a um aumento do uso de redes sociais por crianças e adolescentes. Por mais que isso possa aliviar, em partes, o impacto do isolamento social na saúde mental, uma vez que permite manter a interação social, esses recursos possuem lados negativos nessa população. O uso excessivo pode levar a um vício, comprometendo a rotina do indivíduo; as mídias sociais podem ser um local violento e podem influenciar em problemas de autoimagem, especialmente no público infanto-juvenil (PEDROSA AL, et al., 2020).

\section{A saúde mental no período de isolamento social}

As medidas de restrição realizadas durante a pandemia da COVID-19 possuem um forte impacto na saúde mental da população. A atual conjuntura provocada pela pandemia da COVID-19 possui fatores estressantes que impactam toda sociedade, podendo, inclusive, ser comparada com os impactos psicológicos provocados por desastres naturais e guerras (LIMA SO, et al., 2020).

Acredita-se que a fonte desses impactos psicológicos seja multifatorial, envolvendo fatores como o medo, a incerteza, o estresse, fatores econômicos, a violência doméstica e mudanças nos hábitos diários. $O$ resultado esperado, portanto, é o maior acometimento da saúde psíquica da população, o que pode ser comprovado pelo aumento dos sentimentos de deficiência funcional, tédio, estigma, preocupação, fobia, frustração e raiva (PEDROSA AL, et al., 2020).

Nesse sentido, a saúde mental representa uma das esferas mais abaladas durante a pandemia ao se analisar o impacto nas crianças. Isso ocorre, principalmente, pela forma como a pandemia mudou a maneira como elas se desenvolvem, aprendem, crescem, se comportam, interagem, como lidam com suas emoções, entre outros (SHAH K, et al., 2020). Nesse contexto, estudos demonstram que fatores como quarentena prolongada, medo de infecção, interrupção nas relações sociais, frustração, tédio e perda de entes queridos impactam diretamente na saúde mental dessas crianças e adolescentes (PALACIO-ORTIZ JD, et al., 2020).

Dessa forma, o distanciamento social pode aumentar os problemas relacionados à saúde mental em crianças e adolescentes. A atual situação vivenciada resulta em solidão, uma experiência emocional dolorosa, em que a expectativa social desses jovens não está sendo suprida. Isso já é comprovado por alguns estudos, os quais demonstram que quase um terço dos adolescentes relatam altos níveis de solidão frente à situação atual (LOADES ME, et al., 2020).

Um estudo feito por Chen et al (2020) avaliou 1036 crianças e adolescentes na China, entre seis e quinze anos, durante a quarentena. Segundo a pesquisa, 112 apresentavam depressão, 196 ansiedade e 68 apresentavam ambos. Além disso, outro estudo desenvolvido por Saurabh e Ranjan, na Índia, observou um aumento do sofrimento psicológico, uma vez que esses jovens experimentaram impotência, medo e preocupação durante a quarentena (FIGUEIREDO CS, et al., 2020).

Outro impacto observado na saúde mental dessa parcela da população é o aumento dos sintomas obsessivo-compulsivos, que resultam do medo da contaminação e do estresse. Tais compulsões por limpeza, além da angústia e ansiedade prolongadas tem consequências diretas, tanto no desenvolvimento, como na amplificação de desordens psicológicas (SCHWARTZ-LIFSHITZ M, et al., 2021).

Ademais, é de extrema importância ressaltar que o sistema nervoso central na infância e na adolescência está em uma janela de desenvolvimento vulnerável. Portanto, qualquer exposição a estressores pode levar a consequências de curto e longo prazo, sejam elas cognitivas ou comportamentais (FIGUEIREDO CS, et al., 2020). Dessarte, a vivência frequente de situações que induzam sentimentos de medo, ou mesmo o trauma, pode desencadear repercussões que se estendem até a vida avulta do indivíduo, como pelo desenvolvimento, ou maior propensão, de transtornos de ansiedade, obsessivos-compulsivos, somatização e depressão (MANGUEIRA LFB, et al., 2020).

Diante do exposto, fica claro que as rotinas diárias oferecidas pelas escolas e atividades extracurriculares são fundamentais para que a criança e o adolescente mantenham as atividades físicas, ciclos de sono e relações sociais adequados. Essas atividades são formas de proteção à saúde mental desses indivíduos, de forma que, o distanciamento e todas as restrições sociais acabam contribuindo para quadros ansiosos, depressivos e outros transtornos na saúde mental (COST KT, et al., 2021). 


\section{Impactos do fechamento das escolas e o aprendizado em casa}

Frente ao contexto pandêmico enfrentado pelo mundo, o fechamento das creches, escolas e universidades foi adotado emergencialmente. Para solucionar essa problemática, a solução encontrada fora a adesão do ensino remoto de forma temporária. No entanto, o fechamento dessas instituições repercutiu tanto no contexto educacional, como social, impactando no cotidiano de pais e alunos (JUNIOR JFS e MORAES CCP, 2020).

Dessarte, o fechamento das escolas e a implantação do estudo remoto foi outro desafio enfrentado durante 0 isolamento social. $O$ ensino remoto mostrou-se principalmente desafiador em crianças mais novas (préescolares e séries iniciais) e naquelas com necessidades especiais. A imprescindibilidade de um familiar presente durante as aulas foi maior nesses grupos, o que evidencia o impacto do ensino a distância também nos responsáveis. A falta de interação social, de uma atmosfera de aprendizagem e de atividades ao ar livre foram queixas comuns citadas pelos familiares (MISIRLI O e ERGULEC F, 2021).

Além disso, o processo de aprendizagem foi classificado por pais de crianças com necessidades especiais como estressante e não propício para a aprendizagem (MISIRLI O e ERGULEC F, 2021). Em adolescentes, as dificuldades com o aprendizado em casa envolvem o auto isolamento, dificuldade em ficar em casa, ansiedade, brigas familiares e a preocupação com o déficit educacional (PIGAIANI Y, et al., 2020).

Vale ressaltar que o fechamento das escolas afeta, mais uma vez, as relações sociais das crianças e adolescentes. Essas instituições de ensino são também locais de encontro e interação social, fatores de suma importância no desenvolvimento desses indivíduos. Portanto, estando fechadas, muitas crianças perdem tal referência de contato social, o que prejudica sua aprendizagem e crescimento (JUNIOR JFS e MORAES CCP, 2020).

\section{A alimentação das crianças e adolescentes}

Como era de se esperar, as medidas de contenção da COVID-19 impactaram diretamente na alimentação da população, uma vez que o isolamento e o distanciamento social limitaram a frequência de compras de alimentos, promovendo maior consumo de processados, fast foods e alimentos mais calóricos, por serem de mais fácil aquisição e, muitas vezes, economicamente mais acessíveis (DE SOUZA GC, et al., 2020).

Portanto, no que tange à nutrição de crianças e adolescentes durante o isolamento social, foram evidenciadas pioras na qualidade nutricional das refeições, piora nos hábitos de sono e menor realização de atividade física (PIETROBELLI A, et al., 2020). Esses três fatores estão relacionados com um risco aumentado para o desenvolvimento de obesidade e piora na qualidade de vida. Em contrapartida, a prática de esportes e boa alimentação foram relacionadas com escores de bem-estar maiores durante $o$ isolamento (MORRES ID, et al., 2021).

É importante ressaltar que outros fatores, como a interrupção das aulas presenciais e maior suscetibilidade de afetar a saúde mental desses indivíduos, influenciam diretamente no padrão alimentar e práticas desportivas. Primeiro, sabe-se que o ambiente escolar é essencial para atividades recreativas e práticas esportivas, estimulando a realização de atividades físicas. Segundo, a obesidade possui forte associação com a ansiedade e depressão, fatores os quais, como visto anteriormente, podem muito bem acometer o público infantil durante a pandemia e período de isolamento (DE SOUZA GC, et al., 2020).

Em um estudo italiano que analisou a alimentação de crianças obesas durante o isolamento, foi observado que $\mathrm{o}$ consumo de alimentos processados e carne vermelha aumentou, enquanto o tempo gasto em atividades físicas decaiu (PIETROBELLI A, et al., 2020). Outro estudo, realizado no Brasil, que analisou crianças de até 12 anos, relatou um aumento do tempo de exposição a telas durante a pandemia, sendo maior em meninos do que em meninas. A prática esportiva diminuiu para os dois sexos, porém, meninas realizaram menos atividades físicas em comparação com meninos. A tendência de sedentarismo em ambos os sexos aumenta com o passar da idade (SÁ CSC, et al., 2021; ZHANG X, et al., 2020).

Não só o risco de obesidade, mas também a desnutrição infantil é outro problema intensificado pelo isolamento social e que merece destaque. Apesar da pouca literatura envolvendo o tema, imigrantes, refugiados, populações de baixa renda e crianças com deficiência mental são citados como grupos de risco para desenvolvimento de subnutrição nesse período (ZEMRANI B, et al., 2021). 
Como mencionado anteriormente, a interrupção das aulas presenciais influencia na prática alimentar e podem estar relacionados a esse tema. Isso porque a merenda escolar é uma fonte de alimentação equilibrada e, em alguns casos, é a única refeição de uma criança. Dessa forma, a suspensão das escolas e creches contribui para uma alimentação menos nutritiva para esse público. Ademais, a situação econômica de muitas famílias fora comprometida pelo contexto pandêmico, diminuindo a disponibilidade de compra de alimentos mais nutritivos para crianças e adolescentes de baixa renda (DE SOUZA GC, et al., 2020).

\section{Violência doméstica na pandemia da COVID-19}

Um fator de suma importância que não vem recebendo discussões suficientes acerca do tema na pandemia da COVID-19 é o aumento dos casos de violência doméstica, os quais vêm recrudescendo não só no Brasil, como em diferentes países, a exemplo: China, Reino Unido, Estados Unidos e França (MARQUES ES, et al., 2020). Acredita-se que um dos fatores que mais contribui para esse acontecimento é o maior tempo de convivência familiar durante os tempos de pandemia, onde se encontra boa parte dos agressores (PLATT VB, et al., 2021).

Vale ressaltar que a violência contra esse público é um problema de saúde pública e que envolve não somente a violência física, mas também a negligência, violência sexual ou psicológica. Diante disso, já foi observado que alguns fatores aumentam a suscetibilidade da violência infantil, o que inclui austeridade econômica, desastres e situações de emergências, todas as quais podem se enquadrar na atual pandemia vivenciada (LEVANDOWSKI ML, et al., 2021).

Devido ao distanciamento social e a presença dos pais junto aos filhos por mais tempo, houve um aumento da violência doméstica sofrida pelas crianças, principalmente com relação aos maus tratos e à negligência por causa da instabilidade e incapacidade dos pais de lidarem com a situação. Alguns fatores, como a utilização do mesmo espaço, utilização da mesma internet, aumento nos gastos tanto com comida agravaram o caso (FIGUEIREDO CS, et. al., 2020).

Outros fatores como a baixa disponibilidade de assistência à saúde mental e a grande falta de empregos durante a pandemia fizeram com que os pais se sentissem mais estressados e com menos tempo para pensar em soluções viáveis de como sustentar a família, elevando assim o nível de abuso sofrido pelas crianças. Ademais, a falta de contato social com outros indivíduos, seja nas escolas ou nos parques, diminui a chances de socorro por parte dos jovens (DEOLMI M e PISANI F, 2020).

Além disso, um estudo feito com famílias no Canadá demonstrou que a condição financeira tem significativa influência na questão da violência, pois casas lotadas com poucos recursos tecnológicos e pouco dinheiro para sustentar a família levaram a uma instabilidade emocional e racional dos pais (GADERMANN AC, et al., 2021).

\section{CONSIDERAÇÕES FINAIS}

De acordo com os estudos analisados, observa-se que, embora as crianças e os adolescentes não sejam o grupo mais afetado pelos sintomas da infecção do vírus SARS-COV 2, eles sofrem com as consequências da pandemia e com as medidas necessárias para sua contenção. $O$ isolamento social pode ser apontado como o principal fator que leva às variadas e significativas mudanças no estilo de vida desses indivíduos. $O$ cotidiano das crianças e adolescentes foi afetado de várias formas, como pela interrupção da vida escolar presencial, problemas financeiros e de saúde enfrentados por familiares. Portanto, é de extrema importância atentar-se às crianças nesse momento tão delicado, visando amenizar os impactos da pandemia em sua saúde física e mental.

\section{REFERÊNCIAS}

1. CHEN F, et al. Depression and anxiety among adolescents during COVID-19: A cross-sectional study. Brain, behavior, and immunity, 2020; 88: 36-38.

2. COST KT, et al. Mostly worse, occasionally better: impact of COVID-19 pandemic on the mental health of Canadian children and adolescents. European child \& adolescent psychiatry, 2021; 1-14. 
3. DE SOUSA GC, et al. A Pandemia de COVID-19 e suas repercussões na epidemia da obesidade de crianças e adolescentes. Revista Eletrônica Acervo Saúde, 2020; 12(12): e4743-e4743.

4. DEOLMI M, PISANI F. Psychological and psychiatric impact of COVID-19 pandemic among children and adolescents. Acta Bio Medica: Atenei Parmensis, 2020; 91(4): e2020149

5. DESLANDES SF, COUTINHO T. The intensive use of the internet by children and adolescents in the context of COVID-19 and the risks for self-inflicted violence. Ciencia \& saude coletiva, 2020; 25: 2479-2486.

6. FERNÁNDEZ-ARANDA F, et al. COVID Isolation Eating Scale (CIES): Analysis of the impact of confinement in eating disorders and obesity-A collaborative international study. European Eating Disorders Review, 2020; 28(6): 871-883.

7. FIGUEIREDO CS, et al. COVID-19 pandemic impact on children and adolescents' mental health: Biological, environmental, and social factors. Progress in Neuro-Psychopharmacology and Biological Psychiatry, 2021; 106: 110171.

8. FOLINO CH, et al. A percepção de crianças cariocas sobre a pandemia de COVID-19, SARS-CoV-2 e os vírus em geral. Cadernos de Saúde Pública, 2021; 37: e00304320.

9. GADERMANN AC, et al. Examining the impacts of the COVID-19 pandemic on family mental health in Canada: findings from a national cross-sectional study. BMJ open, 2021; 11(1): e042871.

10. GRABER KM, et al. A rapid review of the impact of quarantine and restricted environments on children's play and the role of play in children's health. Child: care, health and development, 2021; 47(2): 143-153.

11. IMRAN N, et al. Psychological burden of quarantine in children and adolescents: A rapid systematic review and proposed solutions. Pakistan journal of medical sciences, 2020; 36(5): 1106.

12. JUNIOR JFS, MORAES CCP. A COVID-19 e os reflexos sociais do fechamento das escolas. Dialogia, 2020; 36: 128148.

13. LARSEN L, et al. The impact of school closure and social isolation on children in vulnerable families during COVID19: a focus on children's reactions. European child \& adolescent psychiatry, 2021: 1-11.

14. LEVANDOWSKI ML, et al. Impacto do distanciamento social nas notificações de violência contra crianças e adolescentes no Rio Grande do Sul, Brasil. Cadernos de Saúde Pública, 2021; 37(1):e00140020.

15. LIMA SO, et al. Impactos no comportamento e na saúde mental de grupos vulneráveis em época de enfrentamento da infecção COVID-19: revisão narrativa. Revista Eletrônica Acervo Saúde, 2020; 46: e4006-e4006.

16. LOADES ME, et al. Rapid systematic review: the impact of social isolation and loneliness on the mental health of children and adolescents in the context of COVID-19. Journal of the American Academy of Child \& Adolescent Psychiatry, 2020; 59(11): 1218-1239.

17. MANGUEIRA LFB, et al. Saúde mental das crianças e adolescentes em tempos de pandemia: uma revisão narrativa. Revista Eletrônica Acervo Saúde, 2020; 12; 11: e4919-e4919.

18. MARQUES ES, et al. A violência contra mulheres, crianças e adolescentes em tempos de pandemia pela COVID-19: panorama, motivações e formas de enfrentamento. Cadernos de Saúde Pública, 2020; 36: e00074420.

19. MELEGARI MG, et al. Identifying the impact of the confinement of Covid-19 on emotional-mood and behavioural dimensions in children and adolescents with attention deficit hyperactivity disorder (ADHD). Psychiatry research, 2021; 296: 113692

20. MISIRLI O, ERGULEC F. Emergency remote teaching during the COVID-19 pandemic: Parents experiences and perspectives. Education and Information Technologies, 2021: 1-20.

21. MORRES ID, et al. Physical Activity, Sedentariness, Eating Behaviour and Well-Being during a COVID-19 Lockdown Period in Greek Adolescents. Nutrients, 2021; 13(5): 1449.

22. NOGUEIRA-DE-ALMEIDA CA, et al. COVID-19 and obesity in childhood and adolescence: a clinical review, Jornal de Pediatria, 2020; 96: 546-558.

23. PALACIO-ORTIZ JD, et al. Trastornos psiquiátricos en los niños y adolescentes en tiempo de la pandemia por COVID19. Revista Colombiana de Psiquiatría, 2020; 49(4):279-288.

24. PEDROSA AL, et al. Emotional, behavioral, and psychological impact of the COVID-19 pandemic. Frontiers in psychology, 2020, 11: 566212.

25. PIETROBELLI A, et al. Effects of COVID-19 lockdown on lifestyle behaviors in children with obesity living in Verona, Italy: a longitudinal study. Obesity, 2020; 28(8): 1382-1385.

26. PLATT VB, et al. Violence against children and adolescents: notification and alert in times of pandemic. Revista Paulista de Pediatria, 2020, 39: e2020267.

27. PIGAIANI Y, et al. Adolescent Lifestyle Behaviors, Coping Strategies and Subjective Wellbeing during the COVID-19 Pandemic: An Online Student Survey. In: Healthcare. Multidisciplinary Digital Publishing Institute, 2020; 8(4): 472.

28. SÁ CSC, et al. Covid-19 social isolation in brazil: effects on the physical activity routine of families with children. Revista Paulista de Pediatria, 2021, 39: e2020159.

29. SCHWARTZ-LIFSHITZ M, et al. Obsessive compulsive symptoms severity among children and adolescents during COVID-19 first wave in Israel. Journal of obsessive-compulsive and related disorders, 2021; 28: 100610.

30. SEGRE G, et al. Interviewing children: the impact of the COVID-19 quarantine on children's perceived psychological distress and changes in routine. BMC pediatrics, 2021; 21(1): 1-11.

31. SHAH K, et al. Impact of COVID-19 on the Mental Health of Children and Adolescents. Cureus, 2020; 12 (8): e10051.

32. VALERO ALZAGA E, et al. Covid-19 and child health: confinement and its impact according to child professionals. Revista espanola de salud publica, 2020; 94: e202007064.

33. WORLD HEALTH ORGANIZATION (WHO). WHO Director-General's opening remarks at the media briefing on COVID-19-11 March 2020. 2020. Disponível em: https://www.who.int/director-general/speeches/detail/who-directorgeneral-s-opening-remarks-at-the-media-briefing-on-covid-19---11-march-2020. Acesso em: 21 jul. 2021.

34. ZEMRANI B, et al. A hidden side of the COVID-19 pandemic in children: the double burden of undernutrition and overnutrition. International Journal for Equity in Health, 2021; 20(1): 1-4.

35. ZHANG X, et al. Association between physical activity and mood states of children and adolescents in social isolation during the CoViD-19 epidemic. International journal of environmental research and public health, 2020; 17(20): 7666. 